\title{
An asymptotic formula for marginal running coupling constants and universality of loglog corrections
}

\author{
Hisamitsu Mukaida \\ Department of Physics, Saitama Medical College, 981 Kawakado, Moroyama-cho, \\ Iruma-gun, Saitama, 350-0496, Japan \\ E-mail: mukaida@saitama-med.ac.jp
}

\begin{abstract}
Given a two-loop beta function for multiple marginal coupling constants, we derive an asymptotic formula for the running coupling constants driven to an infrared fixed point. It can play an important role in universal loglog corrections to physical quantities.
\end{abstract}

Submitted to: J. Phys. A: Math. Gen.

PACS numbers: 05.10.Cc, 64.60.Ak, 64.60.Fr 


\section{Introduction}

Log and loglog corrections to physical quantities in critical phenomena generally appear in a statistical system at the critical dimension. In the language of renormalization group ( $\mathrm{RG}$ ) [1], those corrections arise from marginally irrelevant coupling constants in the system. As the length scale we are looking at becomes larger, the coupling constants effectively change, obeying a renormalization-group equation (RGE), and approach an infrared fixed point if initial values of the trajectories are on the critical surface. Universality (i.e., property independent of the initial values) of the logarithmic corrections is closely related to the long-distance behavior of the running coupling constants.

Generally, the log and loglog corrections are obtained respectively from the leading and the next-to-leading order of the beta function specifying the RGE. In order to see this, it is instructive to consider the case of a single coupling constant. When the origin is an infrared fixed point, an RGE of a marginal coupling constant $g$ up to the next-to-leading order is generally described as

$$
\frac{\mathrm{d} g}{\mathrm{~d} t}=-a g^{2}+b g^{3}, \quad a>0,
$$

where $t$ is related to a length scale $L$ of RG transformation (RGT) by $t=\ln L$. Here we consider the weak-coupling region between the two fixed points, $0<g<a / b$. It is readily integrated and the solution $g(t)$ satisfies

$$
a t+C=\frac{1}{g(t)}-\frac{b}{a} \ln \left[g(t)\left(\frac{a}{b}-g(t)\right)^{-1}\right]
$$

in this region. The constant $C$ is determined from an initial condition. When $t \rightarrow \infty$, $g(t)$ has the following asymptotic form:

$$
g(t)=\frac{1}{a t}+\frac{b \ln t}{a^{3} t^{2}}+\mathrm{O}\left(t^{-2}\right)
$$

which implies that the first term contributes to a $\ln L$ correction, while the second term to a $\ln \ln L$ correction. A key feature is that the coefficients of $1 / t$ and $\ln t / t^{2}$ are independent of $C$, which leads to universal logarithmic corrections.

Although we can integrate RGE explicitly in the case of a single coupling constant, we cannot generally perform the same procedure in the case of multiple coupling constants. Therefore, it is worthwhile to determine an asymptotic form analogous to (3) in the case of multiple marginal coupling constants, which is the main subject of this paper.

One cannot linearize RGE about a fixed point in the case of marginal coupling constants, which complicates the problem finding an asymptotic form without explicit integration. An algebraic method was found in Refs [2, 3], where the beta function is restricted to the lowest order. Since the lowest-order beta function for marginal coupling constants is homogeneous, the RGE is invariant under scaling transformation [2]. One 
can define another RG transformation to the RGE, thanks to the scale invariance. $\ddagger$ The new RGE generally has a linear term, which allows us to obtain the asymptotic form without explicit integration.

However, we cannot apply the above method when higher orders of the beta function are taken into account because there are no such scale invariances. Hence we need to find an alternative method to remedy the problem for linear terms to vanish.

In the next section, we present a change of variables in the RGE that allows us to apply the linearization. In section 3, we switch from the resultant RGE to an equivalent integral equation, and outline the existence of a unique solution driven to the fixed point. We also give an estimation to the solution. Details of the proof are found in Appendix A. In section 4, using the estimation found in the preceding section, we show a sufficient condition for loglog corrections be universal. A universal asymptotic formula for the solution in the long-distance limit is also derived under the sufficient condition. In section 5, applying our result, we rederive the universal asymptotic formula for the running coupling constants in the classical XY model, as an example. The result is consistent with the original article by Amit et al. [6]. The final section is devoted to summary and discussion.

\section{Changing variables of RGE}

We consider an RGE for marginal coupling constants denoted by $\boldsymbol{g}(t)=\left(g_{1}(t), \ldots, g_{n}(t)\right)$. We regard the space of the coupling constants as the $n$ dimensional Euclidean space $\boldsymbol{R}^{n}$. Suppose that we have obtained the RGE up to the next-to-leading order, which is to say we start with the following RGE

$$
\frac{\mathrm{d} \boldsymbol{g}(t)}{\mathrm{d} t}=\boldsymbol{V}(\boldsymbol{g}(t))+\boldsymbol{F}(\boldsymbol{g}(t)) \text {. }
$$

The leading and the subleading terms of the beta function are described by $\boldsymbol{V}$ and $\boldsymbol{F}$ respectively. It is assumed that they possess the following scaling property:

$$
\boldsymbol{V}(k \boldsymbol{g})=k^{2} \boldsymbol{V}(\boldsymbol{g}), \quad \boldsymbol{F}(k \boldsymbol{g})=k^{3} \boldsymbol{F}(\boldsymbol{g}) .
$$

In general, $\boldsymbol{V}$ and $\boldsymbol{F}$ are obtained as quadratic and cubic polynomials in the coupling constants respectively. The beta function is defined on the whole space $\boldsymbol{R}^{n}$ in this case. However, in some cases, the beta function is obtained in a rational form (e.g., see [7]). For this reason, it is suitable to assume that $\boldsymbol{V}$ and $\boldsymbol{F}$ are defined on some region $E$ in $\boldsymbol{R}^{n} . \oint$

It is a general feature of an RGE of marginal coupling constants that there are no linear terms, which causes difficulty in deriving an asymptotic formula. We introduce new variables to bypass this problem. First we replace $t$ by

$$
u \equiv \frac{1}{\epsilon} \log (\epsilon t+1),
$$

$\ddagger$ A general idea of RG, applied as a tool for asymptotic analysis of non-linear differential equations, is developed in Refs. [4, 5].

$\S$ More precisely, $E$ is open subset of $\boldsymbol{R}^{n}$ whose closure contains the origin. We also assume that $\boldsymbol{V}$ and $\boldsymbol{F}$ belong to $C^{2}(E)$, i.e., their second derivatives exist and are continuous on $E$. 
where $\epsilon$ is a parameter with

$$
0<\epsilon<1 \text {. }
$$

As we will see later, $\epsilon$ is introduced to control an effect of the subleading term $\boldsymbol{F}$. Next we change $\boldsymbol{g}$ to $\boldsymbol{c}$, where

$$
\boldsymbol{g}(t)=\mathrm{e}^{-\epsilon u} \boldsymbol{c}(u) .
$$

The left-hand side of (4) becomes

$$
\begin{aligned}
\frac{\mathrm{d} \boldsymbol{g}(t)}{\mathrm{d} t} & =\mathrm{e}^{-\epsilon u} \frac{\mathrm{d}}{\mathrm{d} u}\left(\mathrm{e}^{-\epsilon u} \boldsymbol{c}(u)\right) \\
& =\mathrm{e}^{-2 \epsilon u}\left(-\epsilon \boldsymbol{c}(u)+\frac{\mathrm{d} \boldsymbol{c}(u)}{\mathrm{d} u}\right),
\end{aligned}
$$

while the right-hand side is

$$
\boldsymbol{V}\left(\mathrm{e}^{-\epsilon u} \boldsymbol{c}\right)+\boldsymbol{F}\left(\mathrm{e}^{-\epsilon u} \boldsymbol{c}\right)=\mathrm{e}^{-2 \epsilon u} \boldsymbol{V}(\boldsymbol{c})+\mathrm{e}^{-3 \epsilon u} \boldsymbol{F}(\boldsymbol{c})
$$

because of the scaling property (5). In this way, the RGE (4) is written as\|

$$
\frac{\mathrm{d} \boldsymbol{c}(u)}{\mathrm{d} u}=\epsilon \boldsymbol{c}(u)+\boldsymbol{V}(\boldsymbol{c}(u))+\mathrm{e}^{-\epsilon u} \boldsymbol{F}(\boldsymbol{c}(u)) .
$$

Now we extract the linear part from the first two terms. We assume that a nontrivial solution $c^{*} \in E$ for

$$
\epsilon \boldsymbol{c}^{*}+\boldsymbol{V}\left(\boldsymbol{c}^{*}\right)=\mathbf{0}
$$

exists. Note that $\boldsymbol{c}^{*}$ is linear in $\epsilon$. In fact, let us introduce $\boldsymbol{a}^{*}$ by

$$
\boldsymbol{c}^{*} \equiv \epsilon \boldsymbol{a}^{*} \text {. }
$$

Because of the scaling property (5), $\boldsymbol{a}^{*}$ is determined by

$$
\boldsymbol{a}^{*}+\boldsymbol{V}\left(\boldsymbol{a}^{*}\right)=\mathbf{0} .
$$

Hence $\boldsymbol{a}^{*}$ is independent of $\epsilon$ and (13) indicates that $\boldsymbol{c}^{*}$ approaches the origin as $\epsilon$ becomes smaller. Therefore, an effect of the subleading term $\boldsymbol{F}$ in a neighborhood of $\boldsymbol{c}^{*}$ is suppressed if we take $\epsilon$ sufficiently small. It plays an important role in showing the existence of a solution for (18) by a contraction map, as we will see in Appendix A.

We analyze (11) in a neighborhood of $\boldsymbol{c}^{*}$. Define

$$
\boldsymbol{b}(u) \equiv \boldsymbol{c}(u)-\boldsymbol{c}^{*}
$$

and write

$$
\boldsymbol{V}(\boldsymbol{c}(u))=\boldsymbol{V}\left(\boldsymbol{c}^{*}\right)+D \boldsymbol{V}\left(\boldsymbol{c}^{*}\right) \boldsymbol{b}(u)+\boldsymbol{v}(\boldsymbol{b}(u)),
$$

where $D \boldsymbol{V}\left(\boldsymbol{c}^{*}\right)$ is the derivative of $\boldsymbol{V}$ at $\boldsymbol{c}^{*}$, which is represented by the $n \times n$ matrix as

$$
D \boldsymbol{V}\left(\boldsymbol{c}^{*}\right)_{i j}=\frac{\partial V_{i}}{\partial c_{j}}\left(\boldsymbol{c}^{*}\right) .
$$

$\|$ We comment that this transformation is generalized in the case where a beta function starts with an $m$ th homogeneous function $(m \geq 2)$. In fact, if we define $u$ and $\boldsymbol{c}(u)$ by $(m-1) \epsilon u=\ln (\epsilon t+1)$ and $\boldsymbol{g}(t)=\mathrm{e}^{-\epsilon u} \boldsymbol{c}(u)$ respectively, we get a similar equation having a linear term. 
The RGE (11) is written as

$$
\frac{\mathrm{d} \boldsymbol{b}(u)}{\mathrm{d} u}=M \boldsymbol{b}(u)+\boldsymbol{H}(u, \boldsymbol{b}(u)),
$$

where

$$
M \equiv \epsilon I_{n}+D \boldsymbol{V}\left(\boldsymbol{c}^{*}\right), \quad \boldsymbol{H}(u, \boldsymbol{b}(u)) \equiv \boldsymbol{v}(\boldsymbol{b}(u))+\mathrm{e}^{-\epsilon u} \boldsymbol{F}\left(\boldsymbol{c}^{*}+\boldsymbol{b}(u)\right)
$$

with $I_{n}$ being the $n \times n$ unit matrix. Further, (5) yields $\Phi$

$$
D \boldsymbol{V}\left(\boldsymbol{c}^{*}\right)=\epsilon D \boldsymbol{V}\left(\boldsymbol{a}^{*}\right) .
$$

Therefore $M$ is linear in $\epsilon$

$$
M=\epsilon\left(I_{n}+D \boldsymbol{V}\left(\boldsymbol{a}^{*}\right)\right) .
$$

To sum up, if we know a solution $\boldsymbol{b}(u)$ for (18), we get a solution for (4) by

$$
\boldsymbol{g}(t)=\mathrm{e}^{-\epsilon u}\left(\boldsymbol{c}^{*}+\boldsymbol{b}(u)\right), \quad u=\frac{1}{\epsilon} \ln (\epsilon t+1) .
$$

\section{Integral equation}

In this section, following reference [8] (Section 2.7), we derive an integral equation satisfied by a solution $\boldsymbol{b}(u)$ for (18) driven to $\mathbf{0}$.

We assume that there are no eigenvalues with zero real part in $M$. Suppose that $M$ has $k$ eigenvalues having a negative real part, and $n-k$ eigenvalues with positive real part. If the positive eigenmodes are fine-tuned to vanish, $|\boldsymbol{b}(u)|$ becomes smaller as $u \rightarrow \infty$. We find from (22) that the corresponding $\boldsymbol{g}(t)$ approaches the origin from the $\boldsymbol{c}^{*}$-direction. In order to show the existence of such solutions, we decompose $M$ into a block diagonal form. Namely,

$$
R^{-1} M R=\left(\begin{array}{cc}
\epsilon P & 0 \\
0 & \epsilon Q
\end{array}\right) \equiv \epsilon \Lambda,
$$

where $\epsilon P$ is a $k \times k$ matrix whose eigenvalues have a negative real part. Similarly, $\epsilon Q$ is an $(n-k) \times(n-k)$ matrix, where its eigenvalues have a positive real part. Note that $P, Q$ and $\Lambda$ are independent of $\epsilon$ because $M$ is linear in $\epsilon$. Define the tilde operation

$$
\tilde{\boldsymbol{x}}=R^{-1} \boldsymbol{x}, \quad \tilde{\boldsymbol{X}}=R^{-1} \circ \boldsymbol{X} \circ R
$$

for a point $\boldsymbol{x} \in E$ and a map $\boldsymbol{X}: E \rightarrow \boldsymbol{R}^{n}$, e.g., $\tilde{\boldsymbol{F}}(\tilde{\boldsymbol{c}})=R^{-1} \boldsymbol{F}(R \tilde{\boldsymbol{c}})=R^{-1} \boldsymbol{F}(\boldsymbol{c})$. The RGE (18) can be written as

$$
\frac{\mathrm{d} \tilde{\boldsymbol{b}}(u)}{\mathrm{d} u}=\epsilon \Lambda \tilde{\boldsymbol{b}}(u)+\tilde{\boldsymbol{H}}(u, \tilde{\boldsymbol{b}}(u)) .
$$

I To prove (20), we use (5) again.

$$
\boldsymbol{V}\left(\boldsymbol{c}^{*}+\boldsymbol{h}\right)=\boldsymbol{V}\left(\boldsymbol{c}^{*}\right)+D \boldsymbol{V}\left(\boldsymbol{c}^{*}\right) \boldsymbol{h}+o(|\boldsymbol{h}|),
$$

while the left-hand side is

$$
\boldsymbol{V}\left(\boldsymbol{c}^{*}+\boldsymbol{h}\right)=\epsilon^{2} \boldsymbol{V}\left(\boldsymbol{a}^{*}+\boldsymbol{h} / \epsilon\right)=\epsilon^{2} \boldsymbol{V}\left(\boldsymbol{a}^{*}\right)+\epsilon D \boldsymbol{V}\left(\boldsymbol{a}^{*}\right) \boldsymbol{h}+o(|\boldsymbol{h}|) .
$$

Comparing the linear term in $\boldsymbol{h}$, we have (20). 
Let

$$
U(u) \equiv\left(\begin{array}{cc}
\mathrm{e}^{P \epsilon u} & 0 \\
0 & 0
\end{array}\right), \quad T(u) \equiv\left(\begin{array}{cc}
0 & 0 \\
0 & \mathrm{e}^{Q \epsilon u}
\end{array}\right)
$$

Then

$$
\frac{\mathrm{d} U}{\mathrm{~d} u}=\epsilon \Lambda U(u), \quad \frac{\mathrm{d} T}{\mathrm{~d} u}=\epsilon \Lambda T(u)
$$

and

$$
\mathrm{e}^{\epsilon \Lambda u}=U(u)+T(u)
$$

We focus on a solution that behaves as $\tilde{\boldsymbol{b}}(u) \rightarrow \mathbf{0}$ as $u \rightarrow \infty$. The integral equation corresponding to it is

$\tilde{\boldsymbol{b}}(u)=U(u) \boldsymbol{p}+\int_{0}^{u} \mathrm{~d} u^{\prime} U\left(u-u^{\prime}\right) \tilde{\boldsymbol{H}}\left(u^{\prime}, \tilde{\boldsymbol{b}}\left(u^{\prime}\right)\right)-\int_{u}^{\infty} \mathrm{d} u^{\prime} T\left(u-u^{\prime}\right) \tilde{\boldsymbol{H}}\left(u^{\prime}, \tilde{\boldsymbol{b}}\left(u^{\prime}\right)\right)$,

where $\boldsymbol{p}=\left(p_{1}, \ldots, p_{k}, 0, \ldots, 0\right)$ specifies an initial condition in the following way:

$$
\begin{aligned}
& \tilde{b}(0)_{i}=p_{i} \quad \text { for } \quad i=1, \ldots, k . \\
& \tilde{b}(0)_{i}=-\left(\int_{0}^{\infty} \mathrm{d} u^{\prime} T\left(-u^{\prime}\right) \tilde{\boldsymbol{H}}\left(u^{\prime}, \tilde{\boldsymbol{b}}\left(u^{\prime}\right)\right)\right)_{i} \quad \text { for } \quad i=k+1, \ldots, n .
\end{aligned}
$$

We can show that (29) has a unique solution if $\epsilon$ and $\boldsymbol{p}$ are sufficiently small. Moreover, we find that the solution satisfies

$$
|\tilde{\boldsymbol{b}}(u)| \leq J \mathrm{e}^{-\alpha \epsilon u}
$$

for some $J>0$. Here $\alpha$ is a positive number, such that $-\alpha$ is strictly greater than the real part of every eigenvalue of $P$. In order to prove (31), we need to extend the usual proof of the stable manifold theorem (see [8]), which is applied to the case of an autonomous system, to the case of the nonautonomous system (18). Details of a proof of (31) are found in Appendix A.

\section{Universal Asymptotic Form of $\tilde{b}(u)$}

In this section, we derive a universal asymptotic form of $\tilde{\boldsymbol{b}}(u)$ by applying (31) to the right-hand side in (29). (Here, "universal" means that the asymptotic form is independent of $\boldsymbol{p}$. )

For this purpose, we give a more concrete form of $P$. Let $\lambda_{l}\left(l=1, \ldots, n_{-}\right)$be the distinct eigenvalues of $P$ with the multiplicity $d_{l}$. We denote by $W_{l}$ the generalized eigenspace associated with $\lambda_{l}$. Clearly, $\operatorname{dim} W_{l}=d_{l}$. Taking an appropriate basis for $\boldsymbol{R}^{n}, P$ is represented as a block diagonal form. Here the $l$ th block $P_{l}$ is a $d_{l} \times d_{l}$ upper triangle matrix whose diagonal components take a common value $\lambda_{l}$. Furthermore, the basis allows us to assume that $N_{l} \equiv P_{l}-\lambda_{l} I_{d_{l}}$ is a nilpotent matrix, namely $N^{\nu_{l}-1} \neq 0$, $N^{\nu_{l}}=0$ for some $1 \leq \nu_{l} \leq d_{l}$. An arbitrary element $\boldsymbol{x} \in \boldsymbol{R}^{n}$ can be decomposed as

$$
\boldsymbol{x}=\sum_{l=1}^{n_{-}} \boldsymbol{x}^{(l)}+\boldsymbol{x}^{(+)}, \quad \boldsymbol{x}^{(l)} \in W_{l},
$$


where $\boldsymbol{x}^{(+)}$is an element of the subspace spanned by the positive eigenmodes of $M$. Applying $U(u)$ to the both sides, we have

$$
U(u) \boldsymbol{x}=\sum_{l=1}^{n_{-}} \mathrm{e}^{\epsilon \lambda_{l} u} \sum_{k=0}^{\nu_{l}-1} \frac{(\epsilon u)^{k} N_{l}^{k}}{k !} \boldsymbol{x}^{(l)},
$$

with the convention $N_{l}^{0}=I_{d_{l}}$ even if $N_{l}$ is the zero matrix.

We can show that ${ }^{+}$

$$
M \boldsymbol{c}^{*}=-\epsilon \boldsymbol{c}^{*},
$$

which implies $P \boldsymbol{c}^{*}=-\boldsymbol{c}^{*}$. Then we set

$$
\lambda_{1}=-1 .
$$

Using (33) and (35), we can estimate the right-hand side of (29). The first term is written as

$$
U(u) \boldsymbol{p}=\sum_{l=1}^{n_{-}} \boldsymbol{G}_{1}^{(l)}(u) \mathrm{e}^{\lambda_{l} \epsilon u},
$$

where $\boldsymbol{G}_{1}^{(l)}(u)$ is a polynomial of degree at most $\nu_{l}-1$. Since (36) explicitly depends on $\boldsymbol{p}$, it is non-universal.

In order to obtain a universal asymptotic form, universal terms should dominate over (36) when $u \rightarrow \infty$. Let us find a condition that such terms appear from the remaining part. The integral containing $U$ in (29) is divided as

$\int_{0}^{u} U\left(u-u^{\prime}\right) \mathrm{e}^{-\epsilon u^{\prime}} \tilde{\boldsymbol{F}}\left(\tilde{\boldsymbol{c}}^{*}\right) \mathrm{d} u^{\prime}+\int_{0}^{u} U\left(u-u^{\prime}\right)\left(\tilde{\boldsymbol{H}}\left(u^{\prime}, \tilde{\boldsymbol{b}}\left(u^{\prime}\right)\right)-\mathrm{e}^{-\epsilon u^{\prime}} \tilde{\boldsymbol{F}}\left(\tilde{\boldsymbol{c}}^{*}\right)\right) \mathrm{d} u^{\prime}$.

Applying (33) to $\tilde{\boldsymbol{F}}\left(\tilde{\boldsymbol{c}}^{*}\right)$, the first integral is easily calculated. It is important to notice that the case of $l=1$ has to be treated separately, because the factor $\exp \left(-\epsilon \lambda_{1} u^{\prime}\right)$ in $U\left(u-u^{\prime}\right)$ cancels $\exp \left(-\epsilon u^{\prime}\right)$ in the integrand. When $l=1$ and $k=\nu_{1}-1$ in (33), the cancellation brings a term proportional to $u^{\nu_{1}} \exp (-\epsilon u)$, which is not contained in $\boldsymbol{G}_{1}^{(l)}(u)$. Writing this explicitly, the integral is expressed as

$$
\int_{0}^{u} U\left(u-u^{\prime}\right) \mathrm{e}^{-\epsilon u^{\prime}} \tilde{\boldsymbol{F}}\left(\tilde{\boldsymbol{c}}^{*}\right) \mathrm{d} u^{\prime}=\frac{(\epsilon u)^{\nu_{1}} \mathrm{e}^{-\epsilon u}}{\epsilon \nu_{1} !} N_{1}^{\nu_{1}-1} \tilde{\boldsymbol{F}}^{(1)}\left(\tilde{\boldsymbol{c}}^{*}\right)+\sum_{l=1}^{n_{-}} \mathrm{e}^{\lambda_{l} \epsilon u} \boldsymbol{G}_{2}^{(l)}(u) .
$$

Here, it is straightforward to check that $\boldsymbol{G}_{2}^{(l)}(u)$ is a polynomial whose degree is at most $\nu_{l}-1$, the same order as (36), so that its universal behavior is obscured by the nonuniversal nature of $U(u) \boldsymbol{p}$. On the other hand, the first term can dominate over (36) in the case when

$$
\Re \lambda_{l}<-1 \quad\left(l=2, \ldots, n_{-}\right) .
$$

+ In order to prove (34), note that

$$
\boldsymbol{V}\left(\boldsymbol{c}^{*}+h \boldsymbol{c}^{*}\right)=\boldsymbol{V}\left(\boldsymbol{c}^{*}\right)+D \boldsymbol{V}\left(\boldsymbol{c}^{*}\right) h \boldsymbol{c}^{*}+o(|h|) .
$$

On the other hand, the left-hand side is equal to

$$
(1+h)^{2} \boldsymbol{V}\left(\boldsymbol{c}^{*}\right)=-\epsilon(1+h)^{2} \boldsymbol{c}^{*}
$$

because of (5) and (12). Comparing the linear term in $h$, we get (34). 
Moreover, as we see in Appendix B, this term is most dominant in the right-hand side of (29) when (39) holds. Thus, we obtain

$$
\tilde{\boldsymbol{b}}(u)=\frac{(\epsilon u)^{\nu_{1}} \mathrm{e}^{-\epsilon u}}{\epsilon \nu_{1} !} N_{1}^{\nu_{1}-1} \tilde{\boldsymbol{F}}^{(1)}\left(\tilde{\boldsymbol{c}}^{*}\right)+\mathrm{O}\left(u^{\nu_{1}-1} \mathrm{e}^{-\epsilon u}\right) .
$$

Let us revert to the original variables by (22). We get, under the condition (39),

$$
\begin{gathered}
\tilde{\boldsymbol{g}}(t)=\frac{1}{\epsilon t+1}\left(\epsilon \tilde{\boldsymbol{a}}^{*}+\frac{\epsilon^{3}(\ln (\epsilon t+1))^{\nu_{1}}}{\epsilon(\epsilon t+1) \nu_{1} !} N_{1}^{\nu_{1}-1} \tilde{\boldsymbol{F}}^{(1)}\left(\tilde{\boldsymbol{a}}^{*}\right)\right)+\mathrm{O}\left(\frac{(\ln (\epsilon t+1))^{\nu_{1}-1}}{(\epsilon t+1)^{2}}\right) \\
=\frac{\tilde{\boldsymbol{a}}^{*}}{t}+\frac{(\ln t)^{\nu_{1}}}{t^{2} \nu_{1} !} N_{1}^{\nu_{1}-1} \tilde{\boldsymbol{F}}^{(1)}\left(\tilde{\boldsymbol{a}}^{*}\right)+\mathrm{O}\left(\frac{(\ln t)^{\nu_{1}-1}}{t^{2}}\right),
\end{gathered}
$$

as $t \rightarrow \infty$. This is the main result of this paper. It is worthwhile to note that the $(\ln t)^{\nu_{1}}$ term appears, which brings about a $(\ln \ln L)^{\nu_{1}}$ correction in general.

The case of $\operatorname{dim} W=1$ The simplest case is that $\operatorname{dim} W_{1}=1$. In this case, using the unit eigenvector $\tilde{\boldsymbol{e}}^{*} \equiv \tilde{\boldsymbol{a}}^{*} /\left|\tilde{\boldsymbol{a}}^{*}\right|$ in $W_{1}$,

$$
\begin{aligned}
\tilde{\boldsymbol{F}}^{(1)}\left(\tilde{\boldsymbol{a}}^{*}\right) & =\left(\tilde{\boldsymbol{F}}\left(\tilde{\boldsymbol{a}}^{*}\right), \tilde{\boldsymbol{e}}^{*}\right) \tilde{\boldsymbol{e}}^{*} \\
N_{1} & =0 \\
\nu_{1} & =1 .
\end{aligned}
$$

Then the result (41) is simplified to

$$
\boldsymbol{g}(t)=\frac{\boldsymbol{a}^{*}}{t}+\frac{\ln t}{t^{2}}\left(\tilde{\boldsymbol{F}}\left(\tilde{\boldsymbol{a}}^{*}\right), \tilde{\boldsymbol{e}}^{*}\right) R \tilde{\boldsymbol{e}}^{*}+\mathrm{O}\left(\frac{1}{t^{2}}\right) .
$$

Note that we have removed ${ }^{\prime \prime}$ from $\tilde{\boldsymbol{g}}$ and $\tilde{\boldsymbol{a}}^{*}$ by applying $R$. Now let us write $R$ as a set of column vectors

$$
R=\left(\boldsymbol{v}_{1}, \ldots, \boldsymbol{v}_{n}\right) .
$$

Similarly, we write $R^{-1}$ in terms of a set of row vectors

$$
R^{-1}=\left(\begin{array}{c}
\boldsymbol{u}_{1} \\
\boldsymbol{u}_{2} \\
\vdots \\
\boldsymbol{u}_{n}
\end{array}\right)
$$

Since $R^{-1} R=I_{n}$, we have

$$
\left(\boldsymbol{v}_{i}, \boldsymbol{u}_{j}\right)=\delta_{i j}
$$

We know that $\boldsymbol{a}^{*}$ is an eigenvector with the eigenvalue -1 , so we can take $\boldsymbol{v}_{1}=\boldsymbol{a}^{*}$. In this case,

$$
\tilde{\boldsymbol{a}}^{*}=R^{-1} \boldsymbol{a}^{*}=\left(\begin{array}{c}
1 \\
0 \\
\vdots \\
0
\end{array}\right)=\tilde{\boldsymbol{e}}^{*}
$$


Therefore

$$
\left(\tilde{\boldsymbol{F}}\left(\tilde{\boldsymbol{a}}^{*}\right), \tilde{\boldsymbol{e}}^{*}\right)=\tilde{F}_{1}\left(\tilde{\boldsymbol{a}}^{*}\right)
$$

where the right-hand side is the first component of $\tilde{\boldsymbol{F}}\left(\tilde{\boldsymbol{a}}^{*}\right)$. Using this convention, the asymptotic behavior (43) is simplified further to

$$
\boldsymbol{g}(t)=\frac{\boldsymbol{a}^{*}}{t}+\frac{\ln t}{t^{2}} \tilde{F}_{1}\left(\tilde{\boldsymbol{a}}^{*}\right) \boldsymbol{a}^{*}+\mathrm{O}\left(\frac{1}{t^{2}}\right) .
$$

\section{Application to the two-dimensional XY model}

In this section, we illustrate our method using the two-dimensional classical XY model [9]. The beta function up to subleading order of this model and the two-point correlation function containing loglog correction are originally derived by Amit et al . [6]. They obtained the asymptotic form of the coupling constants by explicitly integrating the RGE. Here we rederive the asymptotic form within our formulation.

The 2D classical XY model has the following RGE $[6,10]$ :

$$
\begin{aligned}
& \frac{\mathrm{d} g_{1}}{\mathrm{~d} t}=-g_{2}^{2}-B_{1} g_{2}^{2} g_{1} \\
& \frac{\mathrm{d} g_{2}}{\mathrm{~d} t}=-g_{1} g_{2}-A_{1} g_{2}^{3},
\end{aligned}
$$

where $g_{2}>0$ and $2 A_{1}+B_{1}=3 / 2$. Thus

$$
\boldsymbol{V}(\boldsymbol{g})=\left(\begin{array}{c}
-g_{2}^{2} \\
-g_{1} g_{2}
\end{array}\right), \quad \boldsymbol{F}(\boldsymbol{g})=\left(\begin{array}{c}
-B_{1} g_{2}^{2} g_{1} \\
-A_{1} g_{2}^{3}
\end{array}\right) \text {. }
$$

Solving (14), we get a non-trivial solution

$$
\boldsymbol{a}^{*}=\left(\begin{array}{l}
1 \\
1
\end{array}\right)
$$

Inserting this into (21), one finds

$$
M=\left(\begin{array}{cc}
1 & -2 \\
-1 & 0
\end{array}\right) \epsilon .
$$

The eigenvalues and corresponding eigenvectors of $M$ are

$$
-1 \leftrightarrow\left(\begin{array}{l}
1 \\
1
\end{array}\right) \text { and } 2 \leftrightarrow\left(\begin{array}{c}
-2 \\
1
\end{array}\right)
$$

Namely, the space of negative eigenmodes of $M$ is one-dimensional. It indicates that the critical surface along $\boldsymbol{a}^{*}$ is in fact a line. The transformation matrix $R$ and the diagonalized matrix $\Lambda$ are obtained from the eigenvectors and the eigenvalues respectively. The result is

$$
R=\left(\begin{array}{cc}
1 & -2 \\
1 & 1
\end{array}\right), \quad \Lambda=\left(\begin{array}{cc}
-1 & 0 \\
0 & 2
\end{array}\right) \text {. }
$$


The condition (39) is satisfied and $\operatorname{dim} W_{1}=1$ in this example. Furthermore, since we chose $\boldsymbol{a}^{*}$ as the first column in $R,(49)$ is applicable. Using $R$, we compute

$$
\tilde{\boldsymbol{F}}\left(\tilde{\boldsymbol{a}}^{*}\right)=R^{-1} \boldsymbol{F}\left(\boldsymbol{a}^{*}\right)=-\frac{1}{3}\left(\begin{array}{c}
2 A_{1}+B_{1} \\
A_{1}-B_{1}
\end{array}\right) .
$$

From the first component of the above result, we conclude that

$$
\begin{aligned}
\boldsymbol{g}(t) & =\frac{\boldsymbol{a}^{*}}{t}-\frac{1}{3}\left(2 A_{1}+B_{1}\right) \frac{\log t}{t^{2}} \boldsymbol{a}^{*}+\mathrm{O}\left(\frac{1}{t^{2}}\right) \\
& =\frac{\boldsymbol{a}^{*}}{t}-\frac{1}{2} \frac{\log t}{t^{2}} \boldsymbol{a}^{*}+\mathrm{O}\left(\frac{1}{t^{2}}\right)
\end{aligned}
$$

for the critical line. This is consistent with the original result.

\section{Summary and Discussion}

We have obtained an asymptotic formula for multiple marginally irrelevant coupling constants in the case where the two-loop beta functions are known. We first change the variables in the given RGE as (6) and (8). One can extract a linear part $M$ defined in (19) from the resulting differential equation if there is a real solution $\boldsymbol{c}^{*}$ for (12), although the original RGE cannot be linearized. It is assumed that there are no eigenvalues of $M$ with zero real part in the present investigation.

Next we have shown that, if we take $\epsilon$ in (8) to be sufficiently small, there is a $k$ dimensional neighborhood $N$ of $\boldsymbol{c}^{*}$ such that trajectories of the RGE starting in $N$ approach the origin along $\boldsymbol{c}^{*}$, where $k$ is the number of eigenvalues of $M$ with a negative real part. Furthermore, if the eigenvalues of $M$ with a negative real part satisfy the condition (39), the asymptotic formula of $\boldsymbol{g}(t)$ becomes universal and is given by (41).

As we have concretely shown by using the two-dimensional classical XY model, the advantage of this formula is that we do not need to integrate the RGE explicitly.

The non-linearity having the original RGE is changed into solving (12) in our formalism. All quantities appearing in (41) can be computed using simple linear algebra. Thus, our approach can be applicable even though the RGE is too complicated to integrate. Application to such a complicated RGE is a future problem.

Finally, we comment on our previous investigation on this topic. As we mentioned briefly in the introduction, another RGE is derived with respect to the scaling invariance possessed by the leading-order RGE in reference [2], where the asymptotic formula in the in the lowest order is obtained. It is consistent with the present work. The relationship between the linear part of the new RGE and $M$ in (19) is clarified in reference [3]. However, a direct relationship between $M$ and the original RGE was not clear. In this paper, it is found that $M$ naturally comes out in the RGE by the change of variables, which makes it possible to derive the asymptotic formula for a beta function up to the subleading order. The previous formulation can also be applied to deriving the correlation-length exponent of phase transitions in infinite order, which will be extended to the case of the higher-order beta function. 
An asymptotic form for marginal running coupling constants

\section{Acknowledgments}

It is my pleasure to thank C. Itoi for informing me of this attractive problem.

\section{Appendix A. Existence of the solution for (29)}

The purpose of this appendix is to show existence of a unique solution for the integral equation (29) and derive the estimation (31). To this end, we need estimations of $\tilde{\boldsymbol{H}}$, $U$ and $T$. Throughout this appendix, we omit " $"$ for brevity, (e.g., we write $\boldsymbol{b}$ or $\boldsymbol{H}$ instead of $\tilde{\boldsymbol{b}}$ or $\tilde{\boldsymbol{H}}$ respectively. )

\section{Appendix A.1. Lipschitz-type condition for $\boldsymbol{v}$ and $\boldsymbol{f}$}

We first derive a Lipschitz-type condition for $\boldsymbol{v}$ and $\boldsymbol{f}$ defined through the following equation:

$$
\begin{aligned}
& \boldsymbol{V}\left(\boldsymbol{c}^{*}+\boldsymbol{b}\right)=\boldsymbol{V}\left(\boldsymbol{c}^{*}\right)+D \boldsymbol{V}\left(\boldsymbol{c}^{*}\right) \boldsymbol{b}+\boldsymbol{v}(\boldsymbol{b}) \\
& \boldsymbol{F}\left(\boldsymbol{c}^{*}+\boldsymbol{b}\right)=\boldsymbol{F}\left(\boldsymbol{a}^{*}\right)+D \boldsymbol{F}\left(\boldsymbol{a}^{*}\right) \boldsymbol{b}+\boldsymbol{f}(\boldsymbol{b}),
\end{aligned}
$$

which is employed for the estimation of $\boldsymbol{H}$. Let

$$
N_{\eta} \equiv\left\{\boldsymbol{b} ; \boldsymbol{b} \in \boldsymbol{R}^{n},|\boldsymbol{b}|<\eta\right\} .
$$

First we prove that: for any $\xi_{1}, \xi_{2}>0$, there exists a number $\eta>0$ such that

$$
\begin{aligned}
& \left|\boldsymbol{v}\left(\boldsymbol{b}_{1}\right)-\boldsymbol{v}\left(\boldsymbol{b}_{2}\right)\right|<\xi_{1} \epsilon\left|\boldsymbol{b}_{1}-\boldsymbol{b}_{2}\right| \\
& \left|\boldsymbol{f}\left(\boldsymbol{b}_{1}\right)-\boldsymbol{f}\left(\boldsymbol{b}_{2}\right)\right|<\xi_{2} \epsilon\left|\boldsymbol{b}_{1}-\boldsymbol{b}_{2}\right|
\end{aligned}
$$

for $\boldsymbol{b}_{1}, \boldsymbol{b}_{2} \in N_{\epsilon \eta}$ and $0<\epsilon<1$.

Proof: We define $\boldsymbol{v}_{0}$ by the following equation:

$$
\boldsymbol{V}\left(\boldsymbol{a}^{*}+\boldsymbol{b}^{\prime}\right)=\boldsymbol{V}\left(\boldsymbol{a}^{*}\right)+D \boldsymbol{V}\left(\boldsymbol{a}^{*}\right) \boldsymbol{b}^{\prime}+\boldsymbol{v}_{0}\left(\boldsymbol{b}^{\prime}\right) .
$$

For $\boldsymbol{b}_{1}^{\prime}, \boldsymbol{b}_{2}^{\prime}$, let $\boldsymbol{s} \equiv \boldsymbol{b}_{2}^{\prime}-\boldsymbol{b}_{1}^{\prime}$ and define

$$
\boldsymbol{Y}(\theta) \equiv \boldsymbol{v}_{0}\left(\boldsymbol{b}_{1}^{\prime}+\theta \boldsymbol{s}\right) \text {. }
$$

Note that $\boldsymbol{Y}(0)=\boldsymbol{v}_{0}\left(\boldsymbol{b}_{1}^{\prime}\right)$ and $\boldsymbol{Y}(1)=\boldsymbol{v}_{0}\left(\boldsymbol{b}_{2}^{\prime}\right)$. Taking the derivative with respect to $\theta$, we have

$$
\frac{\mathrm{d} \boldsymbol{Y}(\theta)}{\mathrm{d} \theta}=D \boldsymbol{v}_{0}\left(\boldsymbol{b}_{1}^{\prime}+\theta \boldsymbol{s}\right) \boldsymbol{s} .
$$

Integrating from 0 to 1 , we get

$$
\left|\boldsymbol{v}_{0}\left(\boldsymbol{b}_{1}^{\prime}\right)-\boldsymbol{v}_{0}\left(\boldsymbol{b}_{2}^{\prime}\right)\right|=|\boldsymbol{Y}(1)-\boldsymbol{Y}(0)| \leq \int_{0}^{1} \mathrm{~d} \theta\left|D \boldsymbol{v}_{0}\left(\boldsymbol{b}_{1}^{\prime}+\theta \boldsymbol{s}\right)\right||\boldsymbol{s}| .
$$

Since $D \boldsymbol{v}_{0}$ is continuous and $D \boldsymbol{v}_{0}(\mathbf{0})=\mathbf{0}$, for every $\xi_{1}>0$ there exists a number $\eta>0$ such that $\boldsymbol{x} \in N_{\eta}$ implies $\left|D \boldsymbol{v}_{0}(\boldsymbol{x})\right|<\xi_{1}$. For $\boldsymbol{b}_{1}^{\prime}, \boldsymbol{b}_{2}^{\prime} \in N_{\eta}$, since $\boldsymbol{b}_{1}^{\prime}+\theta \boldsymbol{s} \in N_{\eta}$, (A.9) leads to

$$
\left|\boldsymbol{v}_{0}\left(\boldsymbol{b}_{1}^{\prime}\right)-\boldsymbol{v}_{0}\left(\boldsymbol{b}_{2}^{\prime}\right)\right|<\xi_{1}\left|\boldsymbol{b}_{1}^{\prime}-\boldsymbol{b}_{2}^{\prime}\right|
$$


An asymptotic form for marginal running coupling constants

Next we consider estimation in a neighborhood of $\boldsymbol{c}^{*}$. Putting $\boldsymbol{b}=\epsilon \boldsymbol{b}^{\prime}$ and using the scaling property of $\boldsymbol{V}$, we have

$$
\begin{aligned}
\boldsymbol{V}\left(\boldsymbol{c}^{*}+\boldsymbol{b}\right) & =\epsilon^{2} \boldsymbol{V}\left(\boldsymbol{a}^{*}+\boldsymbol{b}^{\prime}\right) \\
& =\epsilon^{2}\left(\boldsymbol{V}\left(\boldsymbol{a}^{*}\right)+D \boldsymbol{V}\left(\boldsymbol{a}^{*}\right) \boldsymbol{b}^{\prime}+\boldsymbol{v}_{0}\left(\boldsymbol{b}^{\prime}\right)\right)
\end{aligned}
$$

Comparing (A.1) and (A.11), we have

$$
\boldsymbol{v}\left(\epsilon \boldsymbol{b}^{\prime}\right)=\epsilon^{2} \boldsymbol{v}_{0}\left(\boldsymbol{b}^{\prime}\right),
$$

which leads to

$$
\begin{aligned}
\left|\boldsymbol{v}\left(\boldsymbol{b}_{1}\right)-\boldsymbol{v}\left(\boldsymbol{b}_{2}\right)\right| & =\epsilon^{2}\left|\boldsymbol{v}_{0}\left(\boldsymbol{b}_{1}^{\prime}\right)-\boldsymbol{v}_{0}\left(\boldsymbol{b}_{2}^{\prime}\right)\right| \\
& <\epsilon^{2} \xi_{1}\left|\boldsymbol{b}_{1}^{\prime}-\boldsymbol{b}_{2}^{\prime}\right| \\
& =\epsilon \xi_{1}\left|\boldsymbol{b}_{1}-\boldsymbol{b}_{2}\right|
\end{aligned}
$$

for $\boldsymbol{b}_{1}, \boldsymbol{b}_{2} \in N_{\epsilon \eta}$. Repeating a similar argument for $\boldsymbol{f}$, we get

$$
\left|\boldsymbol{f}\left(\boldsymbol{b}_{1}\right)-\boldsymbol{f}\left(\boldsymbol{b}_{2}\right)\right|<\epsilon^{2} \xi_{2}\left|\boldsymbol{b}_{1}-\boldsymbol{b}_{2}\right|,
$$

which implies (A.5) because $0<\epsilon<1$.

\section{Appendix A.2. Lipschitz-type condition for $\boldsymbol{H}(u, \boldsymbol{b})$}

Using (A.4) and (A.5), we readily obtain a Lipschitz-type condition for $\boldsymbol{H}$ in the following form: for any $\xi>0$, there exists $\eta>0$ such that

$$
\left|\boldsymbol{H}\left(u, \boldsymbol{b}_{1}\right)-\boldsymbol{H}\left(u, \boldsymbol{b}_{2}\right)\right|<\left(\xi \epsilon+w \epsilon^{2}\right)\left|\boldsymbol{b}_{1}-\boldsymbol{b}_{2}\right|
$$

for all $\boldsymbol{b}_{1}, \boldsymbol{b}_{2} \in N_{\epsilon \eta}$ and $0<\epsilon<1$. Here,

$$
w \equiv\left|D \boldsymbol{F}\left(\boldsymbol{a}^{*}\right)\right| \text {. }
$$

Proof: Recall

$$
\boldsymbol{H}(u, \boldsymbol{b})=\boldsymbol{v}(\boldsymbol{b})+\mathrm{e}^{-\epsilon u} \boldsymbol{F}\left(\boldsymbol{c}^{*}+\boldsymbol{b}\right) .
$$

Then

$$
\begin{aligned}
\left|\boldsymbol{H}\left(u, \boldsymbol{b}_{1}\right)-\boldsymbol{H}\left(u, \boldsymbol{b}_{2}\right)\right| \leq & \left|\boldsymbol{v}\left(\boldsymbol{b}_{1}\right)-\boldsymbol{v}\left(\boldsymbol{b}_{2}\right)\right|+\mathrm{e}^{-\epsilon u}\left|D \boldsymbol{F}\left(\boldsymbol{c}^{*}\right)\left(\boldsymbol{b}_{1}-\boldsymbol{b}_{2}\right)\right| \\
& +\mathrm{e}^{-\epsilon u}\left|\boldsymbol{f}\left(\boldsymbol{b}_{1}\right)-\boldsymbol{f}\left(\boldsymbol{b}_{2}\right)\right| \\
\leq & \left|\boldsymbol{v}\left(\boldsymbol{b}_{1}\right)-\boldsymbol{v}\left(\boldsymbol{b}_{2}\right)\right|+\epsilon^{2} w\left|\boldsymbol{b}_{1}-\boldsymbol{b}_{2}\right| \\
& +\left|\boldsymbol{f}\left(\boldsymbol{b}_{1}\right)-\boldsymbol{f}\left(\boldsymbol{b}_{2}\right)\right|
\end{aligned}
$$

where we have used

$$
\left|D \boldsymbol{F}\left(\boldsymbol{c}^{*}\right)\right|=\epsilon^{2} w .
$$

Using (A.4) and (A.5) for $\xi_{1}=\xi_{2}=\xi / 2$, we get (A.15).

Corollary: if (A.15) holds, then

$$
\left|\boldsymbol{H}\left(u, \boldsymbol{b}_{1}\right)\right|<\left(\xi \epsilon+\epsilon^{2} w\right)\left|\boldsymbol{b}_{1}\right|+\mathrm{e}^{-\alpha \epsilon u} \epsilon^{3}\left|\boldsymbol{F}\left(\boldsymbol{a}^{*}\right)\right|,
$$

where $0<\alpha<1$.

Proof: Setting $\boldsymbol{b}_{2} \equiv \mathbf{0}$ in (A.15), we immediately get

$$
\left|\boldsymbol{H}\left(u, \boldsymbol{b}_{1}\right)-\mathrm{e}^{-\epsilon u} \boldsymbol{F}\left(\boldsymbol{c}^{*}\right)\right|<\left(\xi \epsilon+\epsilon^{2} w\right)\left|\boldsymbol{b}_{1}\right|,
$$

which implies (A.20). 
Appendix A.3. Estimation of $U$ and $T$

Following reference [8] (Section 2.7), we derive: there are positive constants $\alpha, \sigma$ and $K$, such that

$$
\begin{aligned}
& |T(u)|<K \mathrm{e}^{\sigma \epsilon u} \quad(\text { for } u \leq 0) \\
& |U(u)|<K \mathrm{e}^{-(\alpha+\sigma) \epsilon u}(\text { for } u \geq 0),
\end{aligned}
$$

where $0<\alpha<1$.

Proof: Let $\mu_{j}\left(j=1, \ldots, n_{+}\right)$be distinct eigenvalues of $Q$. Here $\Re \mu_{j}>0$ for all $j$. We can choose sufficiently small $\sigma$ and sufficiently large $K$ such that

$$
\begin{aligned}
& \Re \mu_{j}>\sigma>0 \text { for all } j=1, \ldots, n_{+} \\
& |T(u)|<K \mathrm{e}^{\sigma \epsilon u} \text { for all } u \leq 0 .
\end{aligned}
$$

Similarly, let $\lambda_{l}\left(l=1, \ldots, n_{-}\right)$be distinct eigenvalues of $P$. We take positive $\alpha^{\prime}$ satisfying

$$
\Re \lambda_{l}<-\alpha^{\prime}<0
$$

for all $l=1, \ldots, n_{-}$. Then there exists $K^{\prime}>0$ such that

$$
|U(u)|<K^{\prime} \mathrm{e}^{-\alpha^{\prime} \epsilon u}
$$

for all $u \geq 0$. It should be noted that $\alpha^{\prime}<1$ because of (35). Further, if we choose $\sigma$ so small that

$$
\Re \lambda_{l}<-\alpha^{\prime}<-\sigma<0,
$$

then we can write

$$
\alpha^{\prime}=\alpha+\sigma
$$

using some $0<\alpha<1$. Combining (A.23) and (A.25), we obtain (A.22).

Appendix A.4. Existence of a solution

We define the space $\mathcal{C}$ of continuous mappings on $[0, \infty]$ into $\boldsymbol{R}^{n}$ in the following way:

$$
\mathcal{C}=\left\{\boldsymbol{b} ;|\boldsymbol{b}(u)|<\epsilon \eta \mathrm{e}^{-\alpha \epsilon u}, u \geq 0\right\} .
$$

Define the metric on $\mathcal{C}$ by

$$
\rho\left(\boldsymbol{b}_{1}, \boldsymbol{b}_{2}\right)=\sup _{u \geq 0}\left|\left(\boldsymbol{b}_{1}(u)-\boldsymbol{b}_{2}(u)\right) \mathrm{e}^{\alpha \epsilon u}\right|,
$$

for $\boldsymbol{b}_{1}, \boldsymbol{b}_{2} \in \mathcal{C}$. Note that if $\boldsymbol{b} \in \mathcal{C}, \boldsymbol{b}(u) \in N_{\epsilon \eta}$ for all $u \geq 0$. Then according to (A.15), for $\xi>0$, there exists $\eta>0$ such that

$$
\begin{aligned}
\left|\boldsymbol{H}\left(u, \boldsymbol{b}_{1}(u)\right)-\boldsymbol{H}\left(u, \boldsymbol{b}_{2}(u)\right)\right| & <\left(\xi \epsilon+w \epsilon^{2}\right)\left|\boldsymbol{b}_{1}(u)-\boldsymbol{b}_{2}(u)\right| \\
& <\left(\xi \epsilon+w \epsilon^{2}\right) \rho\left(\boldsymbol{b}_{1}, \boldsymbol{b}_{2}\right) \mathrm{e}^{-\alpha \epsilon u}
\end{aligned}
$$

holds for $\boldsymbol{b}_{1}, \boldsymbol{b}_{2} \in \mathcal{C}, u \geq 0$. 
Next we introduce the following mapping $\psi$ on $\mathcal{C}$ :

$\psi(\boldsymbol{b})(u)=U(u) \boldsymbol{p}+\int_{0}^{u} \mathrm{~d} u^{\prime} U\left(u-u^{\prime}\right) \boldsymbol{H}\left(u^{\prime}, \boldsymbol{b}\left(u^{\prime}\right)\right)-\int_{u}^{\infty} \mathrm{d} u^{\prime} T\left(u-u^{\prime}\right) \boldsymbol{H}\left(u^{\prime}, \boldsymbol{b}\left(u^{\prime}\right)\right)$.

We have

$$
\begin{aligned}
\left|\psi\left(\boldsymbol{b}_{1}\right)(u)-\psi\left(\boldsymbol{b}_{2}\right)(u)\right| \leq & K\left(\xi \epsilon+\epsilon w^{2}\right) \rho\left(\boldsymbol{b}_{1}, \boldsymbol{b}_{2}\right) \\
& \times\left(\int_{0}^{u} \mathrm{e}^{-(\alpha+\sigma) \epsilon\left(u-u^{\prime}\right)} \mathrm{e}^{-\alpha \epsilon u^{\prime}} \mathrm{d} u^{\prime}\right. \\
& \left.+\int_{u}^{\infty} \mathrm{e}^{\sigma\left(u-u^{\prime}\right) \epsilon} \mathrm{e}^{-\alpha \epsilon u^{\prime}} \mathrm{d} u^{\prime}\right),
\end{aligned}
$$

according to (A.22) and (A.30). Here the first integration is evaluated as

$$
\int_{0}^{u} \mathrm{e}^{-(\alpha+\sigma) \epsilon\left(u-u^{\prime}\right)} \mathrm{e}^{-\alpha \epsilon u^{\prime}} \mathrm{d} u^{\prime}=\frac{1}{\sigma \epsilon}\left(\mathrm{e}^{-\alpha \epsilon u}-\mathrm{e}^{-(\alpha+\sigma) \epsilon u}\right)<\frac{\mathrm{e}^{-\alpha \epsilon u}}{\sigma \epsilon} .
$$

The second one is

$$
\int_{u}^{\infty} \mathrm{e}^{\sigma\left(u-u^{\prime}\right) \epsilon} \mathrm{e}^{-\alpha \epsilon u^{\prime}} \mathrm{d} u^{\prime}=\frac{1}{(\alpha+\sigma) \epsilon} \mathrm{e}^{-\alpha \epsilon u}<\frac{\mathrm{e}^{-\alpha \epsilon u}}{\sigma \epsilon} .
$$

In this way, we obtain

$$
\rho\left(\psi\left(\boldsymbol{b}_{1}\right), \psi\left(\boldsymbol{b}_{2}\right)\right) \leq \frac{2 K}{\sigma}(\xi+\epsilon w) \rho\left(\boldsymbol{b}_{1}, \boldsymbol{b}_{2}\right)
$$

for $\boldsymbol{b}_{1}, \boldsymbol{b}_{2} \in \mathcal{C}$.

Now we show that $\psi$ is a contraction map on $\mathcal{C}$ into itself if we choose $\xi, \eta, \epsilon$ and $\boldsymbol{p}$ such that the following inequalities hold:

$$
\begin{aligned}
K\left(|\boldsymbol{p}|+\frac{2}{\sigma} \epsilon^{2}\left|\boldsymbol{F}\left(\boldsymbol{a}^{*}\right)\right|\right) & <\frac{1}{2} \epsilon \eta \\
\frac{2 K}{\sigma}(\xi+\epsilon w) & <\frac{1}{2} .
\end{aligned}
$$

It is easy to see that such choice is in fact possible.*

When (A.36) and (A.37) hold, we can show that $\psi$ maps $\mathcal{C}$ into itself. Let us recall (A.20). If $\boldsymbol{b} \in \mathcal{C}$, then

$$
|\boldsymbol{H}(u, \boldsymbol{b}(u))|<\left(\epsilon \eta\left(\xi \epsilon+\epsilon^{2} w\right)+\epsilon^{3}\left|\boldsymbol{F}\left(\boldsymbol{a}^{*}\right)\right|\right) \mathrm{e}^{-\alpha \epsilon u} .
$$

* For example, we first fix $\xi=\xi_{0}$ such that $0<\xi_{0}<\sigma /(4 K)$, which is realized by an appropriate choice of $\eta\left(=\eta_{0}\right)$ according to (A.15). Next we choose $\epsilon=\epsilon_{0}$ satisfying

$$
\epsilon_{0}<\min \left(\frac{\sigma \eta_{0}}{4 K\left|\boldsymbol{F}\left(\boldsymbol{a}^{*}\right)\right|}, \frac{1}{w}\left(\frac{\sigma}{4 K}-\xi_{0}\right)\right)
$$

By construction, $\left(\epsilon_{0}, \xi_{0}\right)$ satisfies (A.37). Furthermore, choosing $\boldsymbol{p}$ such that

$$
0<|\boldsymbol{p}|<\frac{2 \epsilon_{0}\left|\boldsymbol{F}\left(\boldsymbol{a}^{*}\right)\right|}{\sigma}\left(\frac{\sigma \eta_{0}}{4 K\left|\boldsymbol{F}\left(\boldsymbol{a}^{*}\right)\right|}-\epsilon_{0}\right)
$$

we find that (A.36) holds. 
An asymptotic form for marginal running coupling constants

Combining this and the estimations (A.33) and (A.34), (A.31) yields

$$
\begin{aligned}
|\psi(\boldsymbol{b})(u)| & \leq K \mathrm{e}^{-(\alpha+\sigma) \epsilon u}|\boldsymbol{p}|+\left(\epsilon \eta(\xi+\epsilon w)+\epsilon^{2}\left|\boldsymbol{F}\left(\boldsymbol{a}^{*}\right)\right|\right) \frac{2 K}{\sigma} \mathrm{e}^{-\alpha \epsilon u} \\
& <K\left(|\boldsymbol{p}|+\frac{2 K}{\sigma} \epsilon^{2}\left|\boldsymbol{F}\left(\boldsymbol{a}^{*}\right)\right|\right) \mathrm{e}^{-\alpha \epsilon u}+\epsilon \eta(\xi+\epsilon w) \frac{2 K}{\sigma} \mathrm{e}^{-\alpha \epsilon u} \\
& <\epsilon \eta \mathrm{e}^{-\alpha \epsilon u}
\end{aligned}
$$

for all $u \geq 0$. In the last inequality, we have used (A.36) and (A.37). It shows that $\psi(\boldsymbol{b}) \in \mathcal{C}$. Furthermore, (A.35) and (A.37) indicate that $\psi$ is a contraction map.

Thus, existence $\boldsymbol{b} \in \mathcal{C}$ of the solution

$$
\boldsymbol{b}=\psi(\boldsymbol{b})
$$

and its uniqueness follow from the fixed point theorem (see the textbook [11], for example). Since $\boldsymbol{b}$ belongs to $\mathcal{C}$, we find that there is a positive number $J$ such that

$$
|\boldsymbol{b}(u)|<J \mathrm{e}^{-\alpha \epsilon u}
$$

for $u \geq 0$.

\section{Appendix B. Estimation of the remaining terms in (29)}

According to (29) and (36)-(38), it is sufficient to show that

$$
\begin{aligned}
& \left|\int_{0}^{u} U\left(u-u^{\prime}\right)\left(\boldsymbol{H}\left(u^{\prime}, \tilde{\boldsymbol{b}}\left(u^{\prime}\right)\right)-\mathrm{e}^{-\epsilon u} \tilde{\boldsymbol{F}}\left(\tilde{\boldsymbol{c}}^{*}\right)\right) \mathrm{d} u^{\prime}\right|<\sum_{l=0}^{n_{-}} G_{3}^{(l)}(u) \mathrm{e}^{\lambda_{l} \epsilon u} \\
& \left|\int_{u}^{\infty} T\left(u-u^{\prime}\right) \boldsymbol{H}\left(u^{\prime}, \tilde{\boldsymbol{b}}\left(u^{\prime}\right)\right) \mathrm{d} u^{\prime}\right|<B \mathrm{e}^{-\epsilon u},
\end{aligned}
$$

for all $u \geq 0$, in order to complete the derivation of (40). Here $B$ is a positive constant and $G_{3}^{(l)}(u)$ is a polynomial whose degree is at most $\nu_{l}-1$.

Let us first show (B.1). According to (A.2), $\tilde{\boldsymbol{H}}$ is written as

$$
\tilde{\boldsymbol{H}}(u, \tilde{\boldsymbol{b}}(u))=\tilde{\boldsymbol{v}}(\tilde{\boldsymbol{b}}(u))+\mathrm{e}^{-\epsilon u}\left(\tilde{\boldsymbol{F}}\left(\tilde{\boldsymbol{c}}^{*}\right)+D \tilde{\boldsymbol{F}}\left(\tilde{\boldsymbol{c}}^{*}\right) \tilde{\boldsymbol{b}}(u)+\tilde{\boldsymbol{f}}(\tilde{\boldsymbol{b}}(u))\right) .
$$

Since $\tilde{\boldsymbol{V}}, \tilde{\boldsymbol{F}} \in C^{2}(E), \sharp$ the reminders $\tilde{\boldsymbol{v}}, \tilde{\boldsymbol{f}}$ also belong to $C^{2}(E)$. Using the Taylor theorem, we find that there exists a number $\theta(0 \leq \theta \leq 1)$ such that

$$
\tilde{v}_{i}(\tilde{\boldsymbol{b}})=\sum_{j k} \frac{1}{2} \frac{\partial^{2} \tilde{v}_{i}}{\partial \tilde{b}_{j} \partial \tilde{b}_{k}}(\theta \tilde{\boldsymbol{b}}) \tilde{b}_{j} \tilde{b}_{k}
$$

holds. Employing the estimation $(31)$ for $\tilde{\boldsymbol{b}}(u)$, we can show that for sufficiently large $B_{1}$

$$
|\tilde{\boldsymbol{v}}(\tilde{\boldsymbol{b}}(u))|<B_{1} \mathrm{e}^{-2 \alpha \epsilon u} .
$$

A similar estimation holds true for $\tilde{\boldsymbol{f}}$, i.e., for some $B_{2}>0$,

$$
|\tilde{\boldsymbol{f}}(\tilde{\boldsymbol{b}}(u))|<B_{2} \mathrm{e}^{-2 \alpha \epsilon u} .
$$

$\sharp$ see the second footnote in Section 2 . 
If the assumption (39) holds, we can take

$$
\frac{1}{2}<\alpha<1
$$

Further, we choose $\alpha$ such that $2 \alpha \neq-\lambda_{l}$ for all $l=1, \ldots, n_{-}$for later use. Combining the condition (B.7) with (B.5) and (B.6), it follows that there is a number $B_{3}>0$ such that

$$
\begin{aligned}
\left|\tilde{\boldsymbol{H}}(u, \tilde{\boldsymbol{b}}(u))-\mathrm{e}^{-\epsilon u} \tilde{\boldsymbol{F}}\left(\tilde{\boldsymbol{c}}^{*}\right)\right| & <|\tilde{\boldsymbol{v}}(\tilde{\boldsymbol{b}}(u))|+\mathrm{e}^{-\epsilon u}\left(\left|D \tilde{\boldsymbol{F}}\left(\tilde{\boldsymbol{c}}^{*}\right) \tilde{\boldsymbol{b}}(u)\right|+|\tilde{\boldsymbol{f}}(\tilde{\boldsymbol{b}}(u))|\right) \\
& <B_{1} \mathrm{e}^{-2 \alpha \epsilon u}+\left|D \tilde{\boldsymbol{F}}\left(\tilde{\boldsymbol{c}}^{*}\right)\right| \mathrm{e}^{-(1+\alpha) \epsilon u}+B_{2} \mathrm{e}^{(-1-2 \alpha) \epsilon u} \\
& <B_{3} \mathrm{e}^{-2 \alpha \epsilon u}
\end{aligned}
$$

for all $u \geq 0$. Since the same estimation holds for every component projected onto $W_{l}$, we obtain

$$
\begin{aligned}
& \left|U\left(u-u^{\prime}\right)\left(\tilde{\boldsymbol{H}}\left(u^{\prime}, \tilde{\boldsymbol{b}}\left(u^{\prime}\right)\right)-\mathrm{e}^{-\epsilon u} \tilde{\boldsymbol{F}}\left(\tilde{\boldsymbol{c}}^{*}\right)\right)\right| \\
= & \left|\sum_{l=1}^{n_{-}} \mathrm{e}^{\lambda_{l} \epsilon\left(u-u^{\prime}\right)} \sum_{k=0}^{\nu_{l}-1} \frac{\left(\epsilon\left(u-u^{\prime}\right)\right)^{k} N_{l}^{k}}{k !}\left(\tilde{\boldsymbol{H}}^{(l)}\left(u^{\prime}, \tilde{\boldsymbol{b}}\left(u^{\prime}\right)\right)-\mathrm{e}^{-\epsilon u} \tilde{\boldsymbol{F}}^{(l)}\left(\tilde{\boldsymbol{c}}^{*}\right)\right)\right| \\
< & B_{4} \sum_{l=1}^{n_{-}} \mathrm{e}^{\lambda_{l} \epsilon\left(u-u^{\prime}\right)} \sum_{k=0}^{\nu_{l}-1} \frac{\left(\epsilon\left(u-u^{\prime}\right)\right)^{k}}{k !} \mathrm{e}^{-2 \alpha \epsilon u^{\prime}} .
\end{aligned}
$$

Integrating the both sides by $u^{\prime}$ over $[0, u]$, we obtain (B.1).

As for (B.2), we use (B.8). Then we have

$$
\left|\tilde{\boldsymbol{H}}\left(u^{\prime}, \tilde{\boldsymbol{b}}\left(u^{\prime}\right)\right)\right|<\left|\tilde{\boldsymbol{F}}\left(\tilde{\boldsymbol{c}^{*}}\right)\right| \mathrm{e}^{-\epsilon u^{\prime}}+B_{3} \mathrm{e}^{-2 \alpha \epsilon u^{\prime}}<C \mathrm{e}^{-\epsilon u^{\prime}}
$$

for some $C>0$ because we have set $\alpha>1 / 2$. Then the integral is easily estimated as

$\left|\int_{u}^{\infty} \mathrm{d} u^{\prime} T\left(u-u^{\prime}\right) \tilde{\boldsymbol{H}}\left(u^{\prime}, \tilde{\boldsymbol{b}}\left(u^{\prime}\right)\right)\right|<K C \int_{u}^{\infty} \mathrm{d} u^{\prime} \mathrm{e}^{\sigma \epsilon\left(u-u^{\prime}\right)} \mathrm{e}^{-\epsilon u^{\prime}}=\frac{K C}{(\sigma+1) \epsilon} \mathrm{e}^{-\epsilon u}$.

Thus we get (B.2).

\section{References}

[1] Wilson K G and Kogut J 1974 Phys. Rep. 12C 75.

[2] Itoi C and Mukaida H 1999 Phys. Rev. E 603688.

[3] Mukadia H 2004 Phys. Rev. E 70017101.

[4] Chen L -Y, Goldenfeld N and Oono Y 1996 Phys. Rev. E 54376.

[5] Bricmont J and Kupiainen A 1992 Commun. Math. Phys. 150 193; Bricmont J, Kupiainen A and Lin G 1994 Commun. Pure. Appl. Math. 47893.

[6] Amit D J, Goldschmidt Y Y, and Grinstein G G 1980 J. Phys. A: Math. Gen. 13585.

[7] Xing X and Radzihovsky L 2003 Europhys. Lett. 61769.

[8] Perko L 2002 Differential Equations and Dynamical Systems 3rd ed (New York: Springer-Verlag).

[9] Berezinskii V L 1970 Zh. Éksp. Teor. Fiz. 59907 [1971 Sov. Phys. JETP 32 493];

Kosterlitz J M and Thouless D J 1973 J. Phys. C: Solid State Phys. 61181.

[10] J. Balog, and Á. Hegedüs 2000 J. Phys. A: Math. Gen. 336543.

[11] Kolmogorov A N and Fomin S V 1975 Introductory Real Analysis (New York: Dover). 